\title{
Characterisation of Postural Control During A Surf- Like Postural Assessment: Posturographic Analysis
}

\author{
Beatriz Minghelli ${ }^{1 *}$, Filipe Melo ${ }^{2}$, Raul Oliveira ${ }^{2}$ And Carla Nunes ${ }^{3}$ \\ ${ }^{1}$ Research in Education and Community Intervention (RECI), School of Health Jean Piaget Algarve, Piaget Institute, Portugal \\ ${ }^{2}$ Laboratory of Motor Behavior, Faculty of Human Kinetics, University of Lisbon, Portugal \\ ${ }^{3}$ National School of Public Health, NOVA University of Lisbon, Portugal
}

Received: 阱 October 03, 2018 Published: 阱 October 11, 2018

*Corresponding author: Beatriz Minghelli, Research in Education and Community Intervention (RECI), School of Health Jean Piaget Algarve, Piaget Institute, Portugal

\begin{abstract}
Introduction: Surfing practice requires various physical capacities, such as muscular strength and endurance, balance, postural control and neuromuscular coordination. The evaluation of postural control in a surfer is a challenge for sports science and injury prevention researchers. This study evaluated the postural control that surfers perform in a surf-like postural assessment.

Methods: The sample comprised 74 surfers aged between 8-47 years; $83.8 \%$ of the sample were male. Postural control parameters included displacement amplitudes in the anterior-posterior and mid-lateral directions, and length and area of the centre of pressure (CoP) sway. A force platform was positioned on the surfboard, the latter being placed on top of a bozu. Surfers had to perform a task (touching cylinders positioned to their front, right and left) while positioned on top of this platform.

Results: In the group who had up to five years of surf practice, differences in CoP displacement in the mid-lateral direction (38.69mm versus $30.21 \mathrm{~mm})$, the CoP path length $(2079.49 \mathrm{~mm}$ versus $1635.90 \mathrm{~mm})$ and the CoP sway area $(251.36 \mathrm{~mm} 2 \mathrm{versus}$ $161.69 \mathrm{~mm} 2$ ) were statistically significant $(\mathrm{p} \leq 0.05)$, compared with those who had more than five years of surf practice. In the group of surfers who were less than 18 years of age, displacement in the mid-lateral direction ( $38.30 \mathrm{~mm}$ versus $30.54 \mathrm{~mm}$ ) and the length (2059.50mm versus $1652.89 \mathrm{~mm}$ ) were statistically significant $(\mathrm{p} \leq 0.05)$, compared to older surfers.
\end{abstract}

Conclusion: Younger surfers and those who had up to five years of practice showed greater displacement of the CoP. These data are necessary for adopting injury prevention strategies, and specific training.

Keywords: Centre of pressure; Postural sway; Limits of stability; Surfing; Dynamic balance

\section{Introduction}

Surfing is a popular sport practiced at competitive and recreational levels on beaches worldwide [1]. At a competitive level, surfing requires various high intensity physical capacities, such as muscular strength and endurance, balance, postural control and neuromuscular coordination. In addition, athletes are also subject to external factors such as different ocean currents, wind orientation, type of sea floor, size of the waves, water temperature and contact with the board and other surfers, among other factors; these factors require quick and efficient adaptations from the athletes [2].

Nowadays, surfing has become an even more acrobatic and dynamic sport, particularly at competitive levels, increasing the number of injuries acquired while riding waves [3]. The adoption of an incorrect posture due to instability of the board as well as the environment during the course of manoeuvers, generates the ideal scenario for injury to occur. The evaluation of postural control in a surfer is a challenge for sports science and injury prevention researchers. This analysis is necessary so as to assess surfers sensorimotor control, in order to implement injury prevention strategies and to adopt specific training practices out of the water. However, the aquatic environment is hostile for electronic equipment, making it difficult to measure and acquire data concerning postural parameters.

There are several studies [4-6] that developed an electronic solution to determine the kinesiological variables a surfer's body is exposed to (e.g., force and acceleration); this has contributed to the 
understanding of the mechanisms responsible for the occurrence of injuries. However, in these studies no surfers were evaluated. The evaluation of postural control in different profiles of surfers is important to understand if variables such as participating in competitions and exhibiting better execution of techniques, as well as being older (greater maturity) and/or having more years of practice could influence movement control, allowing individuals to perform the manoeuvers more efficiently. This study aimed to characterize, through surf-like postural assessment, the postural control that surfers perform, in order to understand which factors could lead to greater or inferior control of the centre of pressure (CoP).

\section{Methods}

A cross-sectional descriptive survey was used to collect data in this study. This study was approved by the Ethics Committee of the Algarve Regional Health Administration. Written informed consent was obtained from all participants; if under 18 years of age, the informed consent was signed by parents (or the individual who was legally responsible).

\section{Population}

The study population comprised Portuguese surfers residing in southern Portugal. The study included both males and females, and participants were eligible for inclusion if they were 8 years of age or older. All participants agreed to participate voluntarily in the research. To be included, volunteers had to have no injuries at the moment (self-reported), had to have been practicing surfing for at least one year, with at least one training session per week, and had to be available to be present during data acquisition sessions.

\section{Measures}

Measuring instruments included a questionnaire and a force platform. Data were collected in Portimão city (south of Portugal), in different places: Portimão Surf Club, Future Surfing School, Play Surf School and Fisiorider Office, in 2016. Based on a previous study [7], a specific questionnaire was used. This questionnaire was applied to a sample of 101 competitive surfers (85.1\% male; $\mathrm{n}$ $=85$ ), aged 10 to 44 years (19.3 \pm 7.2 years), who participated in the Regional Circuit of the South and Lisbon area in 2015.

The questionnaire included questions about age, gender, position of the feet on the board ("regular" - used the left foot forward and the right foot back, or "goofy" - right foot forward and left foot back), level (recreational or competitive athlete), years of practice, frequency of training per week, participation in competitions in last year, model of boards.

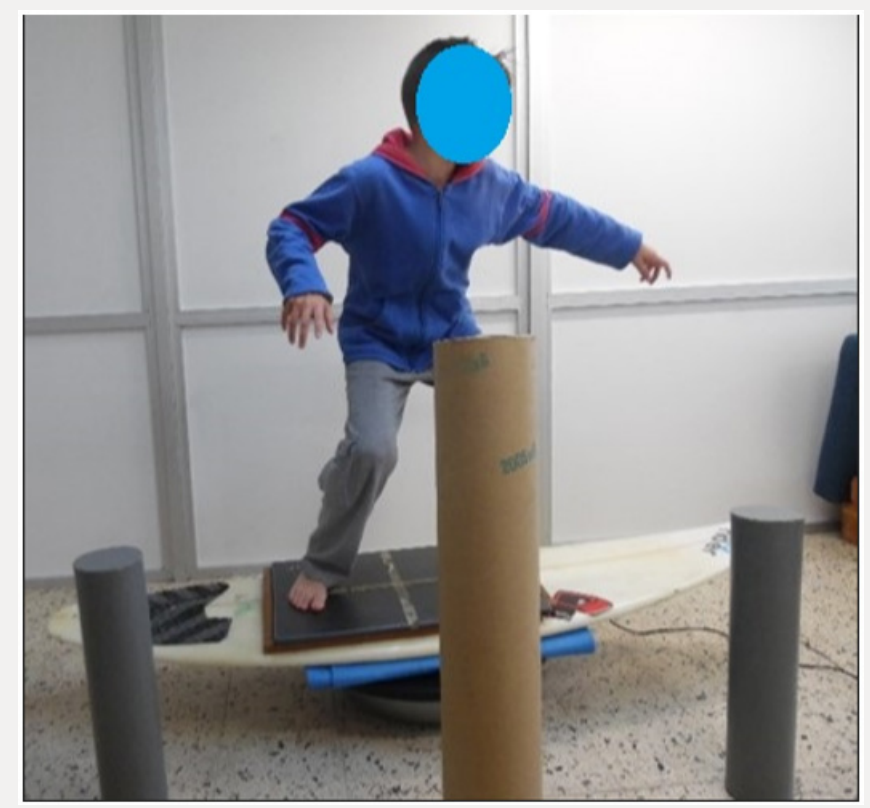

Figure 1: Position of the force plate and surfboard on the top of a Bozu for surfers classified as "regular".

The questionnaire was administered by a researcher with substantial experience in this field. The questionnaire was administered via interview. The researcher did not interfere with the volunteer's opinion or produce biased answers and was able to clarify any possible doubts or questions raised by the volunteers. For the evaluation of postural behaviour, a force platform (Footscan $\AA$ Plate RSscan International, version Balance $2^{\text {nd }}$ generation) was used. Postural balance control was evaluated through the length, amplitude and area travelled by the centre of pressure (CoP) displacement (vertical projection of the centre of gravity on the support base) of a subject standing with bipedal support. The sampling frequency was set at $50 \mathrm{~Hz}$ (number of points/sec). The force platform was positioned on the top of a surfboard, which was located on the top of a Bozu (inverted hemi sphere) to simulate, 
as much as possible, the instability associated with the movement carried out to catch a wave (Figure 1). The orientation of the board took into account the classification of surfers as "regular" or "goofy" (related to the position of the feet).

\section{Description of the Surf-Like Postural Assessment}

Each surfer was requested to stand on the platform while trying to adopt the nearest possible posture they would use to catch a wave, considering that the dimension of the platform was somewhat reduced compared to the distance adopted by some surfers on their boards. All the volunteers performed the test with bare feet and no socks. During the task, surfers were asked to avoid moving their feet. After each instruction from the researcher, surfers had to touch the top of each of the three cylinders positioned in front, right and left of them. The performance of this assessment intended to simulate a position similar to that used by surfers to start manoeuvers, which includes bending the knee and hip joint with angles equal to or greater than $90^{\circ}$ and performing pelvis and trunk rotation movements.

Each task had a total duration of 20 seconds and began as soon as the surfer stood on the force plate in a balanced position. After five seconds, the researcher gave the verbal command to touch the cylinder on the right side with the right hand, for "regular" surfers, and the left cylinder with the left hand, for "goofy" surfers. After that the volunteer was instructed to adopt the starting position. After 10 seconds, each volunteer had to touch the cylinder positioned in front of them with the right hand, if "regular", and left hand if "goofy". After 15 seconds, they had to touch the left cylinder with the right hand, if "regular", and the right cylinder with the left hand, if "goofy". The task was repeated three times with a one-minute interval between each trial (Figures 2\&3). For each volunteer, the trial with the lowest value of postural sway area was selected for data analysis.

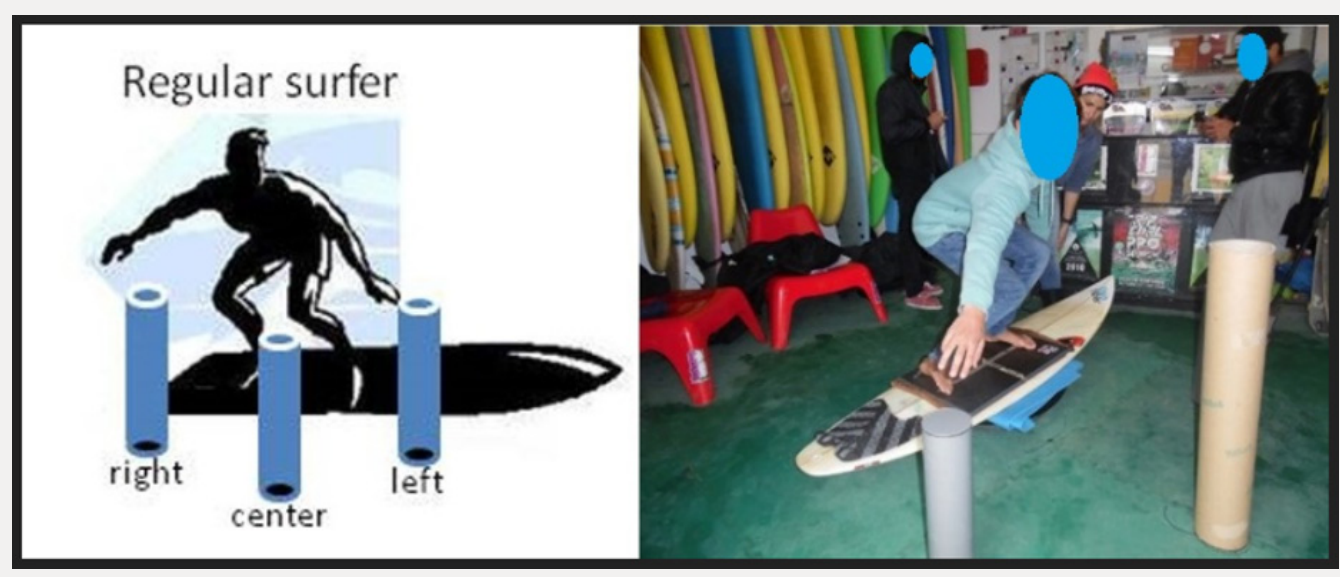

Figure 2: Assessment of a surfer classified as "regular".

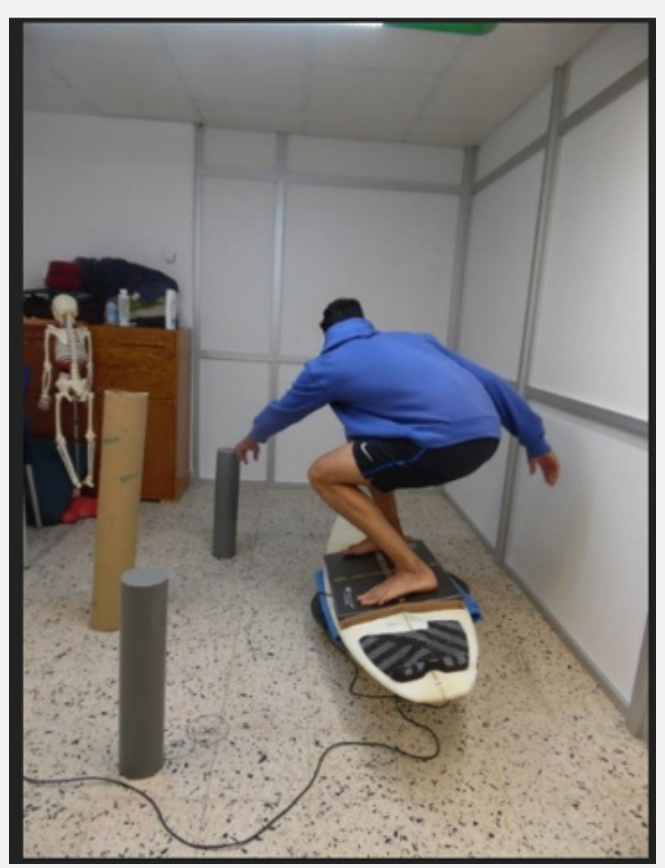

Figure 3: Assessment of a surfer classified as "goofy". 
After each trial, the displacement of the CoP was analysed using RsScan software. This provided data associated with the postural control parameters, including displacement amplitudes in the anterior-posterior and mid-lateral directions, as well as the length and the area of the CoP sway. Displacement in the mid-lateral direction refers to the movements that surfers make towards the nose and tail of the board, and displacement in the anteriorposterior direction refers to the movements that surfers makes from rail to rail.
The positioning of the cylinders took into account the movements required by surfers to perform the maneouvers, and the individual wingspan (Table 1). There was a mark on the bozu that served as a reference point to adjust the location of the cylinders. The position/distance of the cylinders was obtained from previous tests conducted in three athletes with different wingspan values; these athletes were able to perform the task without major difficulties.

Table 1: Position of cylinders in relation to the surfer.

\begin{tabular}{|c|c|c|c|c|}
\hline Surfer Wingspan & $\begin{array}{c}\text { Distance from the front } \\
\text { cylinder }\end{array}$ & $\begin{array}{c}\text { Distance from the right } \\
\text { cylinder }\end{array}$ & $\begin{array}{c}\text { Distance from the left } \\
\text { cylinder }\end{array}$ & $\begin{array}{c}\text { Distance between } \\
\text { cylinders }\end{array}$ \\
\hline $1.40 \mathrm{~m}$ to $1.60 \mathrm{~m}$ & $55 \mathrm{~cm}$ & $60 \mathrm{~cm}$ & $60 \mathrm{~cm}$ & $50 \mathrm{~cm}$ \\
\hline $1.61 \mathrm{~m}$ to $1.80 \mathrm{~m}$ & $65 \mathrm{~cm}$ & $70 \mathrm{~cm}$ & $70 \mathrm{~cm}$ & $65 \mathrm{~cm}$ \\
\hline $1.81 \mathrm{~m}$ to $2.00 \mathrm{~m}$ & $75 \mathrm{~cm}$ & $80 \mathrm{~cm}$ & $80 \mathrm{~cm}$ & $65 \mathrm{~cm}$ \\
\hline
\end{tabular}

\section{Data Analysis}

Descriptive statistics were performed for all variables. The variable age was categorized into two groups, children and adolescents (up to 17 years of age) and adults (aged 18 years and over). The years of practice variable was also divided into two groups, up to five years and over five years of practice. For the application of inferential statistics, the normality of the variables was checked using the Kolmogorov-Smirnov test. According with this, student's t test and the Mann-Whitney $U$ test for two independent samples were used to compare means or distributions of the following variables: CoP displacement amplitude in the anterior-posterior direction, $\mathrm{CoP}$ displacement amplitude in the mid-lateral direction, CoP path length and CoP sway area. In all inferential analyses, the statistical significance level was set at 0.05 . The statistical analysis was performed with the Statistical Package for Social Sciences (SPSS), version 23.0.

\section{Results}

Our sample comprised 74 surfers, 62 (83.8\%) males and 12 (16.2\%) females, aged between 8 and 47 years $(20.04 \pm 10.55$ years), mass between $27.5-96.6 \mathrm{Kg}(58.73 \pm 14.63)$. (male, age $20.92 \pm 11.03$ years, mass $59.54 \pm 14.82 \mathrm{~kg}$; female, age $15.50 \pm 6.09$ years, mass $54.53 \pm 13.38 \mathrm{~kg}$ ). Concerning feet position on the board, $39(52.7 \%)$ surfers were "regular", i.e., used the left foot forward and the right foot back, and 35 (47.3\%) were "goofy", i.e., right foot forward and left foot back.

Twenty-three (31.1\%) surfers has between surfing for 1-2 years, 22 (29.7\%) for 3-5 years, 10 (13.5\%) for 6-7 years, 3 (4.1\%) for $8-9$ years and $16(21.6 \%)$ for more than 10 years. Most surfers ( $\mathrm{n}=38,51.4 \%)$ trained once per week, $13(17.6 \%)$ trained twice a week, $12(16.2 \%)$ three times a week, 8 (10.8\%) four times a week, $2(2.7 \%)$ five times a week, and only one $(1.4 \%)$ surfer trained six times a week.
Forty-three (58.1\%) surfers were recreational and 31 (41.9\%) participated in competitions. Thirty-one $(41.9 \%)$ surfers participated in surfing championships in the last year. Most surfers ( $n=62,83.8 \%$ ), used shortboards; 10 (13.5\%) used longboards and $2(2.7 \%)$ used other types of boards. Seventy-two (97.3\%) volunteers reported that they warm up before surfing practice. Thirty-three $(44.6 \%)$ surfers performed another type of sport two or more times a week. Eighteen (24.3\%) surfers performed a program of prevention.

Descriptive statistics took into account feet position on the board (regular or goofy) and are show in Tables $2 \& 3$ presents the displacement, length and area values as a function of the various categories.

Table 2: Descriptive statistics of data obtained with Rs Scan force platform.

\begin{tabular}{|c|c|c|c|}
\hline Variables & Mean & $\begin{array}{c}\text { Standard } \\
\text { deviation }\end{array}$ & $\begin{array}{c}\text { Minimum- } \\
\text { Maximum }\end{array}$ \\
\hline CoPXmin $(\mathrm{mm})$ & 220.78 & 48.54 & $66-342$ \\
\hline CoPXmax $(\mathrm{mm})$ & 297.99 & 42.85 & $207-391$ \\
\hline CoPXdelta $(\mathrm{mm})$ & 75.76 & 36.45 & $32-279$ \\
\hline CoPYmin $(\mathrm{mm})$ & 139.61 & 19.01 & $82-184$ \\
\hline CoPYmax $(\mathrm{mm})$ & 174.92 & 19.59 & $123-227$ \\
\hline CoPYdelta $(\mathrm{mm})$ & 35.36 & 13.50 & $17-87$ \\
\hline Length $(\mathrm{mm})$ & 1905.65 & 785.89 & $786-4194$ \\
\hline Area $\left(\mathrm{mm}^{2}\right)$ & 216.22 & 235.53 & $51-1922$ \\
\hline
\end{tabular}

CoPXdelta (displacement amplitude in the anterior-posterior direction $)=$ CoPXmax - CoPXmin

CoPYdelta (displacement amplitude in the mid-lateral direction) = CoPYmax - CoPYmin

Length: distance traveled by the center of pressure (CoP)

Area: area related with the oscillation of the centre of pressure $(\mathrm{CoP})$ 
Table 3: Variables data concerning balance control, by group characteristics.

\begin{tabular}{|c|c|c|c|c|c|c|}
\hline \multicolumn{2}{|c|}{ Variables } & Option & Mean & Standard deviation & Median & p-value \\
\hline \multirow{8}{*}{ CoPXdelta } & \multirow{2}{*}{ Practice level } & Competitors (n=25) & 78.97 & 47.29 & 71 & 0.434 \\
\hline & & Free-surfers $(n=43)$ & 73.44 & 26.42 & 68 & \\
\hline & \multirow{2}{*}{ Practice experience } & Until 5 years of practice $(n=45)$ & 79.91 & 41.69 & 71 & 0.322 \\
\hline & & More than 5 years of practice $(n=29)$ & 69.31 & 25.73 & 66 & \\
\hline & \multirow{2}{*}{ Gender } & Male $(\mathrm{n}=62)$ & 78.74 & 38.12 & 72.5 & 0.062 \\
\hline & & Female $(\mathrm{n}=12)$ & 60.33 & 21.23 & 59.5 & \\
\hline & \multirow{2}{*}{ Age group } & Until 17 years of age $(n=46)$ & 78.85 & 40.63 & 70 & 0.489 \\
\hline & & More than 17 years of age $(n=28)$ & 70.68 & 28.25 & 67.5 & \\
\hline \multirow{8}{*}{ CoPYdelta } & \multirow{2}{*}{ Practice level } & Competitors $(\mathrm{n}=25)$ & 36.26 & 15.34 & 33 & 0.884 \\
\hline & & Free-surfers (n=43) & 34.72 & 12.16 & 33 & \\
\hline & \multirow{2}{*}{ Practice experience } & Until 5 years of practice $(n=45)$ & 38.69 & 14.86 & 37 & $0.011^{*}$ \\
\hline & & More than 5 years of practice $(n=29)$ & 30.21 & 9.09 & 29 & \\
\hline & \multirow{2}{*}{ Gender } & Male $(n=62)$ & 35.69 & 13.89 & 33 & 0.741 \\
\hline & & Female $(n=12)$ & 33.67 & 11.69 & 32.5 & \\
\hline & \multirow{2}{*}{ Age group } & Until 17 years of age $(n=46)$ & 38.3 & 13.58 & 37 & $0.005^{*}$ \\
\hline & & More than 17 years of age $(n=28)$ & 30.54 & 12.11 & 29 & \\
\hline \multirow{8}{*}{ Length } & \multirow{2}{*}{ Practice level } & Competitors (n=25) & 1932.26 & 838.99 & 1850 & 0.746 \\
\hline & & Free-surfers $(\mathrm{n}=43)$ & 1886.47 & 754.85 & 1737 & \\
\hline & \multirow{2}{*}{ Practice experience } & Until 5 years of practice $(n=45)$ & 2079.49 & 852.15 & 1940 & $0.029 *$ \\
\hline & & More than 5 years of practice $(n=29)$ & 1635.90 & 587.54 & 1427 & \\
\hline & \multirow{2}{*}{ Gender } & Male $(n=62)$ & 1935.26 & 824.92 & 1715 & 0.628 \\
\hline & & Female $(\mathrm{n}=12)$ & 1752.67 & 542.85 & 1853.5 & \\
\hline & \multirow{2}{*}{ Age group } & Until 17 years of age $(n=46)$ & 2059.50 & 826.59 & 1912.5 & $0.034^{*}$ \\
\hline & & More than 17 years of age $(n=28)$ & 1652.89 & 651.57 & 1432.5 & \\
\hline \multirow{8}{*}{ Area } & \multirow{2}{*}{ Practice level } & Competitors (n=25) & 248.48 & 339.94 & 151 & 0.391 \\
\hline & & Free-surfers $(n=43)$ & 192.95 & 112.07 & 147 & \\
\hline & \multirow{2}{*}{ Practice experience } & Until 5 years of practice $(n=45)$ & 251.36 & 284.57 & 205 & $0.033^{*}$ \\
\hline & & More than 5 years of practice $(n=29)$ & 161.69 & 110.95 & 128 & \\
\hline & \multirow{2}{*}{ Gender } & Male $(\mathrm{n}=62)$ & 226.42 & 251.97 & 152 & 0.232 \\
\hline & & Female $(\mathrm{n}=12)$ & 163.5 & 111.61 & 123.5 & \\
\hline & \multirow{2}{*}{ Age group } & Until 17 years of age $(n=46)$ & 239.52 & 278.74 & 188 & 0.116 \\
\hline & & More than 17 years of age $(n=28)$ & 177.93 & 134.32 & 125 & \\
\hline
\end{tabular}

\section{Discussion}

This study aimed to characterise postural control among surfers, using a simulated scenario (surf-like postural assessment) out of water that was as similar as possible to some movements that surfers perform in the water.

There are other studies that have developed simulated environments to quantify the movements that occur when surfing in water, but none, as far we know, test these instruments in the water. For example, Bona et al. [4] explored an electronic solution to dynamically measure centre of pressure and surfboard movement, with two matrices composed of 24 force sensors and an inertial measurement unit controlled by an ATEMEGA1280 microcontroller. However, this system was only tested using an unstable structure.
Junior et al. [5] constructed an electronic system (M32C Mitsubishi single-chip microcomputer based) embedded in a surfboard for measurement of displacement, but the system is still being tested in the laboratory.

The results of the present study revealed that displacement in the anterior-posterior and mid-lateral directions, the length and the area of the CoP were higher in the competitors group, compared to the free-surfers group, in the group of surfers who had been surfing for less than five years, compared to those with more years of practice, in the group who were under 18 years of age, compared to older volunteers, and in male surfers, compared to females. However, for most groups, these differences were not statistically significant. For the "years of practice" groups, there was a statistically significant difference between the groups in 
the displacement in the mid-lateral direction, as well as the length and area. Similarly, for the "age" groups, there was a statistically significant between the groups in the displacement in the midlateral direction and the length.

To perform the various manoeuvres and to stay on the board, surfers are always changing their CoP position (vertical projection of the center of gravity) and altering their balance and weight distribution between both feet. Balance can be defined as the ability to maintain the vertical projection of the center of gravity on its support base with minimum oscillation and maximum stability. The ability to control balance in standing posture is based on a complex interaction between somatosensory, vestibular and visual functions, as well as coordination between the different body segments [8]. In the case of surfing, balance control is highly affected by the nature of the task, the environmental conditions and the available sensory information [2].

Surfers with less than five years' experience and those less than 18 years of age, even if participating in competitions, require greater improvement of the correct technique of body weight transfer during performance of manoeuvers. As such, to correctly perform a certain manoeuver, surfers have to transfer body weight to the front foot, as well as keep greater control of body posture, that is, knees in valgus and flexed position, in order to maintain or increase speed and obtain better directional control of the board. Since this control requires time and practice, the younger and less experienced surfers have not yet developed a correct technique for performing weight transfer on the board when subjected to an imbalance. This promotes greater displacement of the centre of pressure, especially in activities that require weight displacement in the mid-lateral direction. Surfers have to displace weight towards the front foot, which is only acquired by an improvement in technique, and thus greater sensorimotor control, with time (years of practice) and age. In addition, bone growth is still taking place in this age group (under 18 years of age), as such, the bone segments may still be disproportionate to each other, leading to changes in postural balance.

Although no statistically significant difference was observed, we found that surfers participating in competitions (compared with free-surfers) had greater displacement of the centre of pressure during our experimental task. Competitors are forced to perform manoeuvers with a greater degree of difficulty in order to obtain a higher score in competitions. This forces them to move their centre of gravity further in order to gain speed and perform these kinds of manoeuvers; such a task implies increased instability, followed by a regain of postural control. In other words, during the surf session, surfers have to perform complex movements during wave riding, which include changing the surfboard's orientation and velocity using rotation of the upper body and/or the feet position in order to control the surfboard's direction.
Concerning gender, it was observed that male surfers had higher displacement of the centre of pressure as compared to women, although this difference was not statistically significant. This result may be due to the fact that male surfers perform their manoeuvers with greater intensity (force and aggressiveness) in order to spread a greater amount of water, resulting in better and more spectacular manoeuvers. Better stability in surfing can be associated with lower displacement of the centre of pressure (shorter length) and area; however, as already mentioned, to obtain greater speed and complexity in the accomplishment of manoeuvers, this situation should be reversed. That is, surfers generate greater imbalances and then quickly achieve stabilization to accomplish more demanding manoeuvers.

As mentioned before, research investigating postural behaviour in surfers, in or out of the water, are scarce, so our experimental scenario tried to simulate, as close as possible, the surfing environment in terms of foot and balance stimulation. We believe that, for the reasons already mentioned, competitive athletes seek to perform using the limits of stability. However, since our task did not truly correspond to the tasks performed when the surfers are in the water and are subjected to several external factors, we consider it important to perform studies that evaluate surfer's postural control in the water. In addition, future studies should explore homogeneous samples, such as a sample of professional surfers, to evaluate the postural behaviour of these athletes in a more competitive and realistic environment. These studies should use specific electronic equipment in association with a kinematic analysis of movement, even if each wave and manoeuver is not reproducible.

\section{Conclusion}

The data from this study revealed that surfers with less than five years of experience, and those under 18 years of age, showed greater displacement of the centre of pressure, especially in activities that require weight displacement in the mid-lateral direction. This is probably because these surfers require greater development of the correct technique of body weight transfer during performance of these complex manoeouvers; this technique can be acquired with more years surfing experience and with a greater level of biological maturation (age). These types of studies, which evaluate the postural control of surfers, are necessary for the implementation of injury prevention strategies, and for adopting specific training out of the water.

\section{Acknowledgment}

Filipe Costa, physiotherapist in the surf area, for having loaned the material for the evaluations, and helped in the development of the task; Future Surfing School, and Portimão Surf Club, for having made available the facilities for task evaluation; Swell-Algarve, by the disclosure of the study; and all the athletes who participated in the study. 


\section{References}

1. Mendez-Villanueva A, Bishop D, Hamer P (2006) Activity profile of world-class professional surfers during competition: A case study. Journal of Strength and Conditioning Research 20(3): 477-482.

2. Moraes G, Guimarães A, Gomes A (2013) Analysis of the prevalence of injuries in the Paraná coast surfers. Acta Ortop Bras 21(4): 213-218.

3. Nathanson A (2013) Surfing Injuries. In: Mei-Dan O, Carmont M (Eds); Adventure and Extreme Sports Injuries. Springer-Verlag, London, pp. 143-172.

4. Bona D, Marques M, Borgonovo-Santos M, Correia M (2014) Monitoring of plantar forces and surfboard's movement: Alternative to understand the injuries mechanism. In: Medical Measurements and Applications (MeMeA), Paper presented at the meeting of IEEE International Symposium, Lisboa.
5. Junior N, Kauffmann P, Santos M, Borges L, Dias J, et al. (2007) Paper presented at the meeting of XXV ISBS Symposium, Ouro Preto, Brazil.

6. Lestrade K, Guérard S, Lanusse P, Viot Ph (2015) Biomechanics of surfing: Development and validation of an instrumented surfboard to measure surfboard kinetics. Paper presented at $33^{\text {rd }}$ International Conference on Biomechanics in Sports.

7. Minghelli B, Costa F, Moreira P, Vicente M (2016) Preventive Physiotherapy applied to Portuguese surf athletes: Association in improving performance and reducing the number of injuries. Int J Sports Exerc Med 2(3): 043.

8. Emery C (2003) Is there a clinical standing balance measurement appropriate for use in sports medicine? A review of the literature. J Sci Med Sport 6(4): 492-504.

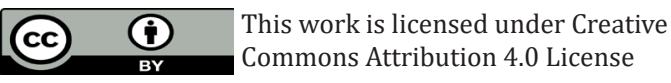

To Submit Your Article Click Here: Submit Article

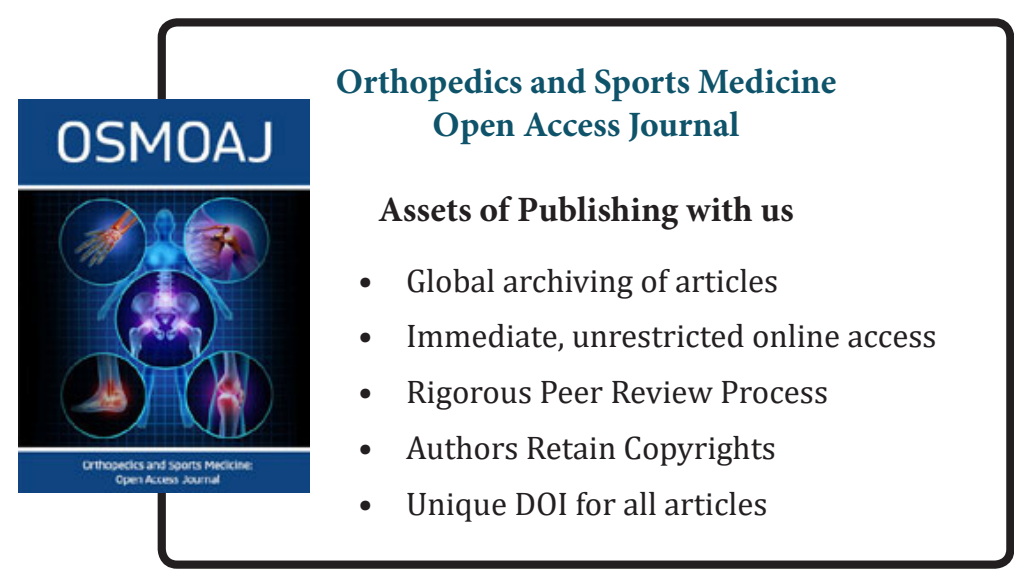

